\title{
Foundation of Z-numbers and Engineering Applications
}

\author{
Sina Razvarz ${ }^{1[0000-0003-1549-7307]}$, Filipe Hernandez Rodriguz ${ }^{2}$ \\ Raheleh Jafari ${ }^{3[0000-0001-7298-2363]}$, and Alexander Gegov 4[0000-0002-6166-296X] \\ ${ }^{1}$ Departamento de Control Automatico, CINVESTAV-IPN (National Polytechnic Institute) \\ Mexico City 07360, Mexico \\ srazvarz@yahoo.com \\ ${ }^{2}$ School of Engineering and science,Tecnologico de Monterrey,Prol.Juan de la Bar- \\ rera 1241, Saltillo25270, Mexico \\ Felipe.hdz@tec.mx \\ ${ }^{3}$ School of design, University of Leeds, LS2 9TJ, Leeds, UK \\ r.jafari@leeds.ac.uk \\ ${ }^{4}$ School of Computing, University of Portsmouth, Buckingham Building, Portsmouth PO1 \\ $3 \mathrm{HE}, \mathrm{UK}$ \\ alexander.gegov@port.ac.uk
}

\begin{abstract}
. decision-making problems in economics, engineering, risk assessment, forecasting, manufacturing, design and process analysis are naturally determined by vagueness as well as partial reliability. Generating a formalism for dealing with Z-numbers is essential to handle these kinds of information. In this paper, the applications of $Z$-numbers in engineering are studied. The most current researches in the area of $Z$ - numbers are covered in this work. Since some engineers have successfully applied Z-numbers, detailed discussions are supplied to stimulate future investigations.
\end{abstract}

Keywords: Z-Number, Decision-Making, Reliability, Engineering Applications

\section{Introduction}

mainly for the system with a mathematical design which is hard to obtain [1-13]. Fuzzy set theory is considered as an efficient technique to deal with uncertainty. It has been effectively implemented to resolve multi-criteria decision making problems. Nevertheless, the main issue in decision-making is the reliability of the information associated with the procedure.

Real-world information is often vague and also is normally represented in natural language. Moreover, this information is usually partially reliable and a degree of reliability is likewise expressed in natural language. To establish a basis to handle this vagueness and partial reliability, in [14] the idea of Z-number is developed. The Z-number is defined as an ordered pair $Z=(U, V)$ of fuzzy numbers utilized to explain a value of a random variable $X$, such that $\mathrm{U}$ is taken to be a fuzzy constraint on values of $X$, also $V$ is taken to be a reliability which is a value of probability rate of $U$. Z-numbers are extensively utilized in a wide variety of implementations in different fields [15-22]. In 
[23] a novel analytic hierarchy process technique is proposed based on the Z-numbers for handling the linguistic decision-making problems. In [24] an arithmetic technique to discrete Z-numbers is suggested. In [25] an arithmetic technique to continuous Znumbers is presented.

To demonstrate the usage of Z-numbers in engineering and also for providing a basis for future investigation, a literature review of the Z-numbers in engineering is conducted in this paper. This article remaining sections are organized into three Sections. Section 2 presents some basic definitions related to Z-numbers. In Section 3 the applications of Z-number in engineering are given. Conclusions are included in Section 4.

\section{Mathematical preliminaries}

We consider the following definitions.

Definition 1. Suppose $c$ is:

1) normal, there is $\varsigma_{0} \in \Re$ where $c\left(\varsigma_{0}\right)=1$,

2)convex, $c(\beta \varsigma+(1-\beta) \varsigma) \geq \min \{c(\varsigma), c(\varrho)\}, \forall \varsigma, \varrho \in \Re, \forall \beta \in[0,1]$,

3)upper semi-continuous on $\mathfrak{R}, c(\varsigma) \leq c\left(\varsigma_{0}\right)+\varepsilon, \forall \varsigma \in N\left(\varsigma_{0}\right), \forall \varsigma_{0} \in \mathfrak{R}, \forall \varepsilon>$ $0, N\left(\varsigma_{0}\right)$ is a neighborhood,

4) $c^{+}=\{\varsigma \in \Re, c(\varsigma)>0\}$ is compact, so $c$ is a fuzzy variable, $c \in E: \Re \rightarrow[0,1]$.

The fuzzy variable $\mathrm{c}$ is demonstrated as

$$
c=(\underline{c}, \bar{c})
$$

in which $\mathrm{c}$ is the lower-bound variable also, $\mathrm{c}$ is the upper-bound variable.

Definition 2. The Z-number is made up of two components $Z=[c(s), p]$. The first component $c(\varsigma)$ is the restriction on a real-valued uncertain variable $\varsigma$. The second component $p$ is a measure of the reliability of $c . p$ can be reliability, strength of belief, probability or possibility. The Z-number can be stated as $Z^{+}$- number, in a case $c(\varsigma)$ be a fuzzy number also $p$ be the probability distribution of $\varsigma$. If $c(\varsigma)$, as well as p, are fuzzy numbers, the Z-number can be stated as $Z^{-}$-number.

The $Z^{+}$-number contains more information when compared with the $Z^{-}$- number. In this paper, the definition of $Z^{+}$-number is utilized, i.e., $Z=[c, p], c$ is a fuzzy number also, $\mathrm{p}$ is a probability distribution.

The most common membership functions which define the fuzzy numbers are the triangular function

$$
\mu_{c}=H(s, u, v)=\left\{\begin{array}{l}
\frac{\varsigma-\mathrm{s}}{u-s} s \leq \varsigma \leq \mathrm{u} \\
\frac{v-\varsigma}{v-u} u \leq \varsigma \leq v
\end{array} \text { otherwise } \mu_{c}=0\right.
$$

and trapezoidal function 


$$
\mu_{c}=H(s, u, v, w)=\left\{\begin{array}{l}
\frac{\varsigma-\mathrm{s}}{u-s} s \leq \varsigma \leq \mathrm{u} \\
\frac{w-\varsigma}{w-v} v \leq \varsigma \leq w \\
1 u \leq \varsigma \leq v
\end{array} \text { otherwise } \mu_{c}=0\right.
$$

If $c$ represents a fuzzy event in $\Re$, the real line, then the probability measure can be stated as

$$
P(c)=\int_{\Re} \mu_{c}(\varsigma) p(\varsigma) d \varsigma
$$

where $p$ is the probability density of $\varsigma$. For discrete Z-numbers we have

$$
P(c)=\sum_{l=1}^{n} \mu_{c}\left(s_{l}\right) p\left(s_{l}\right)
$$

Definition 4. The $\alpha$-level of the Z-number $Z=(c, p)$ is defined as follows

$$
[Z]^{\alpha}=\left([c]^{\alpha},[p]^{\alpha}\right)
$$

where $0<\alpha \leq 1$. $[p]^{\alpha}$ is computed by the Nguyen's theorem

$$
[p]^{\alpha}=p\left([c]^{\alpha}\right)=p\left(\left[\underline{c}^{\alpha}, \bar{c}^{\alpha}\right]\right)=\left[\underline{P}^{\alpha}, \bar{P}^{\alpha}\right]
$$

where $p\left([c]^{\alpha}\right)=\left\{p(\varsigma) \mid \varsigma \in[c]^{\alpha}\right\}$. Therefore, $[Z]^{\alpha}$ is stated as

$$
[Z]^{\alpha}=\left(\underline{Z}^{\alpha}, \bar{Z}^{\alpha}\right)=\left(\left(\underline{c}^{\alpha}, \underline{P}^{\alpha}\right),\left(\bar{c}^{\alpha}, \bar{P}^{\alpha}\right)\right)
$$

where $\underline{P}^{\alpha}=\underline{c}^{\alpha} p\left(\underline{\varsigma}^{\alpha}\right), \bar{P}^{\alpha}=\bar{c}^{\alpha} p\left(\bar{\varsigma}^{\alpha}\right),\left[\varsigma_{l}\right]^{\alpha}=\left(\underline{\varsigma}_{l}^{\alpha},{\overline{\varsigma_{l}}}^{\alpha}\right)$.

The same as with the fuzzy numbers [24], three main operations are defined for the Znumbers: $\oplus, \ominus$ and $\odot$, which indicate sum, subtract and multiply respectively.

Suppose $Z_{1}=\left(c_{1}, p_{1}\right)$ as well as $Z_{2}=\left(c_{2}, p_{2}\right)$ are two discrete Z-numbers expressing the uncertain variables $\varsigma_{1}$ and $\varsigma_{2}$, so, $\sum_{l=1}^{n} p_{1}\left(\varsigma_{1 l}\right)=1, \sum_{l=1}^{n} p_{2}\left(\varsigma_{2 l}\right)=1$.

The following operation is defined

$$
Z_{12}=Z_{1} * Z_{2}=\left(c_{1} * c_{2}, p_{1} * p_{2}\right)
$$

Where $* \in\{\oplus, \ominus, \odot\}$

The operations used for the fuzzy numbers $\left[c_{1}\right]^{\alpha}=\left[c_{11}^{\alpha}, c_{12}^{\alpha}\right]$ and $\left[c_{2}\right]^{\alpha}=\left[c_{21}^{\alpha}, c_{22}^{\alpha}\right]$ are stated as [26],

$$
\begin{aligned}
{\left[c_{1} \oplus c_{2}\right]^{\alpha} } & =\left[c_{1}\right]^{\alpha}+\left[c_{2}\right]^{\alpha}=\left[c_{11}^{\alpha}+c_{21}^{\alpha}, c_{12}^{\alpha}+c_{22}^{\alpha}\right] \\
{\left[c_{1} \ominus c_{2}\right]^{\alpha} } & =\left[c_{1}\right]^{\alpha}-\left[c_{2}\right]^{\alpha}=\left[c_{11}^{\alpha}-c_{21}^{\alpha}, c_{12}^{\alpha}-c_{22}^{\alpha}\right] \\
{\left[c_{1} \odot c_{2}\right]^{\alpha} } & =\left(\begin{array}{l}
\min \left\{c_{11}^{\alpha} c_{21}^{\alpha}, c_{11}^{\alpha} c_{22}^{\alpha}, c_{12}^{\alpha} c_{21}^{\alpha}, c_{12}^{\alpha} c_{22}^{\alpha}\right\} \\
\max \left\{c_{11}^{\alpha} c_{21}^{\alpha}, c_{11}^{\alpha} c_{22}^{\alpha}, c_{12}^{\alpha} c_{21}^{\alpha}, c_{12}^{\alpha} c_{22}^{\alpha}\right\}
\end{array}\right)
\end{aligned}
$$

For the discrete probability distributions, the following relation is defined for all $p_{1} *$ $p_{2}$ operations

$$
p_{1} * p_{2}=\sum_{l} p_{1}\left(\varsigma_{1}, \iota\right) p_{2}\left(\varsigma_{2,(n-\imath)}\right)=p_{12}(\varsigma)
$$

The Hukuhara difference is defined as [17], 


$$
\begin{gathered}
Z_{1} \ominus_{H} Z_{2}=Z_{12} \\
Z_{1}=Z_{2} \oplus Z_{12}
\end{gathered}
$$

In a case that $Z_{1} \ominus_{H} Z_{2}$ exists, the $\alpha$-level can be defined as

$$
\left[Z_{1} \ominus_{H} Z_{2}\right]^{\alpha}=\left[\underline{Z}_{1}^{\alpha}-\underline{Z}_{2}^{\alpha}, \bar{Z}_{1}^{\alpha}-\bar{Z}_{2}^{\alpha}\right]
$$

$\alpha$ Clearly, $Z_{1} \ominus_{H} Z_{1}=0, Z_{1} \ominus Z_{1} \neq 0$.

Moreover, the generalized Hukuhara difference is defined as [22],

$$
Z_{1} \ominus_{g H} Z_{2}=Z_{12} \Leftrightarrow\left\{\begin{array}{l}
\text { 1) } Z_{1}=Z_{2} \oplus Z_{12} \\
\text { 2) } Z_{2}=Z_{1} \oplus(-1) Z_{12}
\end{array}\right.
$$

By taking into consideration the $\alpha$-level, we have $\left[Z_{1} \ominus_{H} Z_{2}\right]^{\alpha}$ $=\left[\min \left\{\underline{Z}_{1}^{\alpha}-\underline{Z}_{2}^{\alpha}, \bar{Z}_{1}^{\alpha}-\bar{Z}_{2}^{\alpha}\right\}, \max \left\{\underline{Z}_{1}^{\alpha}-\underline{Z}_{2}^{\alpha}, \bar{Z}_{1}^{\alpha}-\bar{Z}_{2}^{\alpha}\right\}\right]$ and If also, if $Z_{1} \ominus_{H} Z_{2}$ exists, $Z_{1} \ominus_{H} Z_{2}=Z_{1} \ominus_{g H} Z_{2}$. The conditions for the existence of $Z_{12}=Z_{1} \ominus_{g H} Z_{2} \in E$ are

$$
\begin{gathered}
\text { 1) }\left\{\begin{array}{c}
\underline{Z}_{12}^{\alpha}=\underline{Z}_{1}^{\alpha}-\underline{Z}_{2}^{\alpha} \text { and } \bar{Z}_{12}^{\alpha}=\bar{Z}_{1}^{\alpha}-\bar{Z}_{2}^{\alpha} \\
\text { with } \underline{Z}_{12}^{\alpha} \text { increa sing, } \bar{Z}_{12}^{\alpha} \text { decrea sing, } \underline{Z}_{12}^{\alpha} \leq \bar{Z}_{12}^{\alpha}
\end{array}\right. \\
\text { 2) }\left\{\begin{array}{c}
\underline{Z}_{12}^{\alpha}=\bar{Z}_{1}^{\alpha}-\bar{Z}_{2}^{\alpha} \text { and } \bar{Z}_{12}^{\alpha}=\underline{Z}_{1}^{\alpha}-\underline{Z}_{2}^{\alpha} \\
\text { with } \underline{Z}_{12}^{\alpha} \text { increa sing, }, \bar{Z}_{12}^{\alpha} \text { decrea sing, } \underline{Z}_{12}^{\alpha} \leq \bar{Z}_{12}^{\alpha}
\end{array}\right.
\end{gathered}
$$

where $\forall \alpha \in[0,1]$.

Suppose $\mathrm{c}$ is a triangular function, the absolute value of the $\mathrm{Z}$-number $\mathrm{Z}=(\mathrm{c}, \mathrm{p})$ is defined as

$$
|\mathrm{Z}(\varsigma)|=\left(\left|\mathrm{s}_{1}\right|+\left|\mathrm{u}_{1}\right|+\left|\mathrm{v}_{1}\right|, \mathrm{p}\left(\left|\mathrm{s}_{2}\right|+\left|\mathrm{u}_{2}\right|+\left|\mathrm{v}_{2}\right|\right)\right)
$$

Let $c_{1}$ as well as $c_{2}$ are triangular functions, the supremum metric for $Z$-numbers $\mathrm{Z}_{1}=\left(\mathrm{c}_{1}, \mathrm{p}_{1}\right)$ and $\mathrm{Z}_{2}=\left(\mathrm{c}_{2}, \mathrm{p}_{2}\right)$ is expressed as

$$
\mathrm{D}\left(\mathrm{Z}_{1}, \mathrm{Z}_{2}\right)=\mathrm{d}\left(\mathrm{c}_{1}, \mathrm{c}_{2}\right)+\mathrm{d}\left(\mathrm{p}_{1}, \mathrm{p}_{2}\right)
$$

where $d(\cdot, \cdot)$ is the supremum metrics for fuzzy sets [22]. $D\left(Z_{1}, Z_{2}\right)$ has the below mentioned properties, 


$$
\begin{aligned}
& \mathrm{D}\left(Z_{1}+\mathrm{Z}, \mathrm{Z}_{2}+\mathrm{Z}\right)=\mathrm{D}\left(\mathrm{Z}_{1}, \mathrm{Z}_{2}\right) \\
& \mathrm{D}\left(\mathrm{Z}_{2}, \mathrm{Z}_{1}\right)=\mathrm{D}\left(\mathrm{Z}_{1}, \mathrm{Z}_{2}\right) \\
& \mathrm{D}\left(\mathrm{b} \mathrm{Z}_{1}, \mathrm{k} Z_{2}\right)=\mathrm{b} \mid \mathrm{D}\left(\mathrm{Z}_{1}, \mathrm{Z}_{2}\right) \\
& \mathrm{D}\left(\mathrm{Z}_{1}, \mathrm{Z}_{2}\right) \leq \mathrm{D}\left(\mathrm{Z}_{1}, \mathrm{Z}\right)+\mathrm{D}\left(\mathrm{Z}, \mathrm{Z}_{2}\right)
\end{aligned}
$$

where $b \in \Re, Z=(c, p)$ is $Z$-number, also $c$ is triangle function.

Definition 5. Suppose $Z$ is the space of $Z$-numbers. The $\alpha-$ level of the $Z$-number valued function $\mathrm{H}:[0, \mathrm{~s}] \rightarrow \mathrm{Z}$ is defined as

$$
\mathrm{H}(\mathrm{c}, \alpha)=[\underline{\mathrm{H}}(\mathrm{c}, \alpha), \overline{\mathrm{H}}(\mathrm{c}, \alpha)]
$$

where $\mathrm{c} \in \mathrm{Z}$, for each $\alpha \in[0,1]$.

Using the definition of Generalized Hukuhara difference, the $\mathrm{gH}$-derivative of $\mathrm{H}$ at $\mathrm{c}_{0}$ is defined as

$$
\frac{\mathrm{d}}{\mathrm{dt}} \mathrm{H}\left(\mathrm{c}_{0}\right)=\lim _{\zeta \rightarrow 0} \frac{1}{\zeta}\left[\mathrm{H}\left(\mathrm{c}_{0}+\zeta\right) \ominus_{g H} \mathrm{H}\left(\mathrm{c}_{0}\right)\right]
$$

In (25), $\mathrm{H}\left(\mathrm{c}_{0}+\zeta\right)$ as well as $\mathrm{H}\left(\mathrm{c}_{0}\right)$ represent symmetric pattern with $\mathrm{Z}_{1}$ and $\mathrm{Z}_{2}$ respectively given in (19).

In this book chapter, the aim of the modeling is to find the Z-number coefficient $a_{1}$ for the below fuzzy equation

$$
\mathrm{w}_{\mathrm{r}}^{*}=\sum_{\mathrm{l}=1}^{\gamma} \mathrm{a}_{\mathrm{l}} \mathrm{f}_{\mathrm{l}}\left(\vartheta_{\mathrm{r}}\right)
$$

in such a way that the output of the plant $\mathrm{w}_{\mathrm{r}}$ approaches to the desired output $\mathrm{w}_{\mathrm{r}}^{*}$.

Definition 6. The distance between two Z-numbers, $\psi, \phi \in \mathrm{Z}$, is described as the Hausdorff metric $\mathrm{D}_{\mathrm{H}}[\psi, \phi]$,

$$
\begin{gathered}
\mathrm{D}_{\mathrm{H}}[\psi, \phi]=\max \left\{\operatorname { s u p } _ { ( \mathrm { a } _ { 1 } , \mathrm { c } _ { 1 } ) \in \psi } \operatorname { i n f } _ { ( \mathrm { a } _ { 2 } , \mathrm { c } _ { 2 } ) \in \phi } \left(\mathrm{d}\left(\mathrm{a}_{1}, \mathrm{a}_{2}\right)\right.\right. \\
\left.+\mathrm{d}\left(\mathrm{c}_{1}, \mathrm{c}_{2}\right)\right), \sup _{\left(\mathrm{a}_{1}, \mathrm{c}_{1}\right) \in \phi} \inf _{\left(\mathrm{a}_{2}, \mathrm{c}_{2}\right) \in \psi}\left(\mathrm{d}\left(\mathrm{a}_{1}, \mathrm{a}_{2}\right)+\mathrm{d}\left(\mathrm{c}_{1}, \mathrm{c}_{2}\right)\right\}
\end{gathered}
$$

$\mathrm{d}(\mathrm{a}, \mathrm{c})$ is the supremum metrics defined for fuzzy sets.

Lemma 1. Let $\varpi \subset Z$ is a compact set, in this case, $\varpi$ is uniformly support-bounded, i.e. there exists a compact set $\Psi \subset \mathfrak{R}$, in such a way that $\forall \psi \in \varpi$,

$$
\operatorname{Supp}(\psi) \subset \Psi \text {. }
$$

Lemma 2. Suppose $\psi, \phi \in Z$, also $\alpha \in(0,1]$, in this case the following is concluded:

(i) if $\mathrm{f}, \mathrm{g}: \mathfrak{R} \rightarrow \mathfrak{R}$ are continuous, $[\mathrm{f}(\psi)]^{\alpha}=\mathrm{f}\left(\left[\psi^{\alpha}\right]\right)$ holds; 
(ii) if $\mathrm{f}: \mathfrak{R} \rightarrow \mathfrak{R}$ are continuous, so $\mathrm{f}(\operatorname{Supp}(\psi))=\operatorname{Supp}(\mathrm{f}(\psi))$.

proof. As (i) concludes from (Yang \& Gao, 1993), so just (ii) will be proved. Initially, $\overline{f\left(D_{1}\right)}=f\left(\bar{D}_{1}\right)$ is shown for $D_{1} \subset \Re$. As $f\left(D_{1}\right) \subset f\left(\bar{D}_{1}\right)$, also $f\left(\bar{D}_{1}\right)$ is closed by the continuity of $f$, therefore, $\overline{f\left(D_{1}\right)} \subset \mathrm{f}\left(\bar{D}_{1}\right)$. Also, for randomly given $\theta \in f\left(\bar{D}_{1}\right)$, there exists a sequence $\left\{a_{n} \mid n \in N\right\} \subset \Re, a \in \mathfrak{R}$, in such a way that $a_{n} \rightarrow a(n \rightarrow+\infty)$, $\theta=f(a)$. Since $f$ is continues so, $\lim _{n \rightarrow+\infty} f\left(a_{n}\right)=f(a)=\theta$

However, $\mathrm{f}\left(\mathrm{a}_{\mathrm{n}}\right) \in \mathrm{f}\left(\mathrm{D}_{1}\right)$, therefore, $\theta \in \overline{\mathrm{f}\left(\mathrm{D}_{1}\right)}$. So $\mathrm{f}\left(\overline{\mathrm{D}}_{1}\right) \subset \overline{\mathrm{f}\left(\mathrm{D}_{1}\right)}$. Hence $\overline{\mathrm{f}\left(\mathrm{D}_{1}\right)}=\mathrm{f}\left(\overline{\mathrm{D}}_{1}\right)$

Since,

$$
\begin{gathered}
\operatorname{Supp}(\mathrm{f}(\psi))=\overline{\{\theta \in \mathfrak{R} \mid(\mathrm{f}(\psi))(\theta)>0\}} \\
\mathrm{f}(\operatorname{Supp}(\psi))=\mathrm{f}(\overline{\{\mathrm{a} \in \mathfrak{R} \mid \psi(\mathrm{a})>0\}})
\end{gathered}
$$

the following is concluded

$$
\begin{gathered}
\mathrm{f}(\operatorname{Supp}(\psi))=\overline{\mathrm{f}(\{\mathrm{a} \in \mathfrak{R} \mid \psi(\mathrm{a})>0\})} \\
=\overline{\{\mathrm{f}(\mathrm{a}) \in \mathfrak{R} \mid \psi(\mathrm{a})>0\}}
\end{gathered}
$$

As, $\{\theta \in \mathfrak{R} \mid(\mathrm{f}(\psi))(\theta)>0\}=\{\mathrm{f}(\mathrm{a}) \mid \psi(\mathrm{a})>0\}$, so

$$
\operatorname{Supp}(\mathrm{f}(\psi))=\mathrm{f}(\operatorname{Supp}(\psi))
$$

Lemma 3. Suppose $G \subset \Re$ is a compact set, also $f_{1}$ as well as $f_{2}$ are continuous on $\mathrm{G}, \zeta>0$, in addition

$$
\left|\mathrm{f}_{1}(\mathrm{a})-\mathrm{f}_{2}(\mathrm{a})\right|<\zeta, \quad \forall \mathrm{a} \in \mathrm{G}
$$

Hence $\left|\sup _{a \in G_{1}} f_{1}(a)-\sup _{a \in G_{1}} f_{2}(a)\right|<\zeta$ is valid for each compact set $G_{1} \subset G$.

Theorem 1. Suppose f $: \mathfrak{R} \rightarrow \mathfrak{R}$ is a continuous function, so for each compact set $\varpi \subset \mathrm{Z}_{0}$ (the set of all the bounded Z-number set) also $\chi>0$, there exists $\mathrm{a}_{1} \in \mathrm{Z}_{0}$, $\imath=1,2, \ldots, \gamma$, in such a manner that

$$
\mathrm{d}\left(\mathrm{f}(\tilde{\vartheta}), \sum_{\mathrm{l}=1}^{\gamma} \mathrm{f}_{\mathrm{l}}(\vartheta) \mathrm{a}_{\mathrm{l}}\right)<\chi \quad \forall \vartheta \in \varpi, \forall \tilde{\vartheta} \in \mathfrak{R}
$$

in which $\chi$ is a finite number.

Theorem 2. If $\mathrm{f}: \mathfrak{R} \rightarrow \mathfrak{R}$ is a continuous function, so for each compact set $\varpi \subset \mathrm{Z}_{0}$, $\tau>0$ also arbitrary $\varepsilon>0$, there exists $a_{1} \in Z_{0}, \quad \imath=1,2, \ldots, \gamma$, in such a way that

$$
\mathrm{d}\left(\mathrm{f}(\vartheta), \sum_{\mathrm{l}=1}^{\gamma} \mathrm{f}_{\mathrm{l}}(\vartheta) \mathrm{a}_{\mathrm{l}}\right)<\tau, \quad \forall \vartheta \in \varpi
$$


in which, $\tau$ is termed as a finite number. The lower as well as the upper limits of the $\alpha$-level set of Z-number function approach to $\tau$, however the center approaches to $\varepsilon$.

Theorem 3. Suppose $\varpi \subset \mathrm{Z}_{0}$ is compact, also $\Psi$ is the corresponding compact set of $\varpi$ and $\mathrm{f}, \hat{\mathrm{f}}: \mathfrak{R} \rightarrow \mathfrak{R}$ are the continuous functions that,

$$
|\mathrm{f}(\vartheta)-\hat{\mathrm{f}}(\vartheta)|<\zeta, \quad \forall \vartheta \in \Psi
$$

where $\zeta>0$. So, $\forall \psi_{1} \in \varpi$, d $\left(\mathrm{f}\left(\psi_{1}\right)-\hat{\mathrm{f}}\left(\psi_{1}\right)\right) \leq \zeta$.

\section{$3 \quad$ Applications of $\mathrm{Z}$-number in engineering}

Multi-sensor data fusion, a functional simulation of the process related to the decisionmaking executed by the human brain, has obtained a high growth level over the past few years across industries. In [27] a new technique with the combination of the Znumber and Dempster Shafer evidence theory is suggested for modeling as well as processing the uncertainties in a sensor data fusion system. In that technique, the Z-number is utilized for modeling the fuzziness as well as the reliability of the sensor data also the Dempster-Shafer evidence theory is utilized for fusing the uncertain information of Z-numbers. The major benefits of the suggested technique in [27] are that it produces a perfect robust degree of re- liability to the sensor data, also the supplementary information of multi-sensors decreases the uncertainty of the fault detection, hence increasing the reliability of fault identification. The process of fault detection with the technique proposed in [27] is shown in Fig. 1.

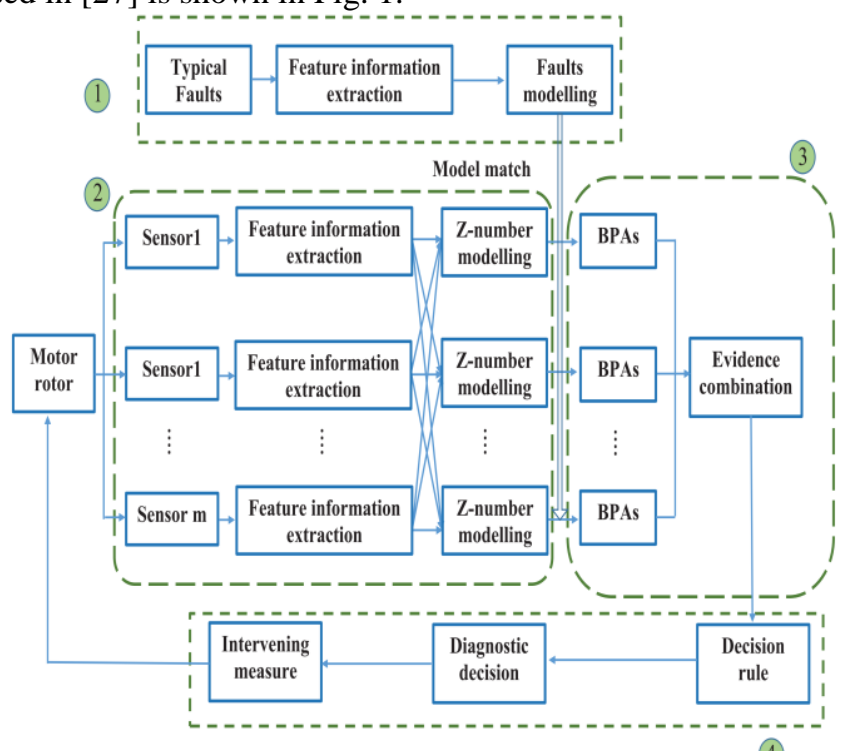

(4)

Fig.1. The process of fault detection. 
In [28] Bernstein neural network is used for finding the Z-number solutions of fuzzy differential equations. In [28] a generalized model of a tank system is proposed which is in the form of the fuzzy differential equation, see Fig. 2. The tank model is described as

$$
\frac{d}{d t} y(t)=-\frac{1}{C J} y(t)+\frac{k}{C}
$$

where $K$ is inflow disturbances of the tank that generates vibration in liquid level $y, J$ is the flow obstruction which can be curbed utilizing the valve, also $C$ is the crosssection of the tank.

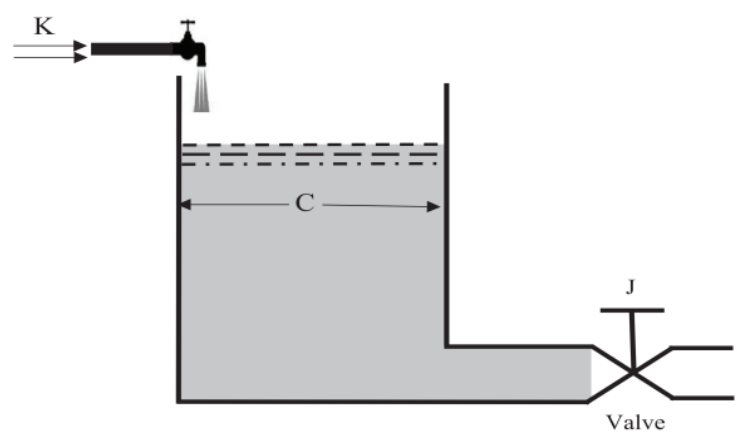

Fig.2. Liquid tank system.

The project management has become a significant approach nearly in all fields of human activity. Every project is temporary by nature and also it has the beginning and finish dates. In [29], the capabilities of the Z-numbers in the improvement of the state of risk evaluation is studied.

In [30], fuzzy equations with Z-numbers are used to design the fuzzy controller. In [30], a heat source by insulating materials is proposed which is in the form of the fuzzy equation, see Fig. 3. The insulating materials center is considered to be the source of heat. The model is described as

$$
\frac{A}{K_{1}} \oplus \frac{B}{K_{2}}=\frac{C}{K_{3}} \oplus \frac{D}{K_{4}} \oplus R
$$




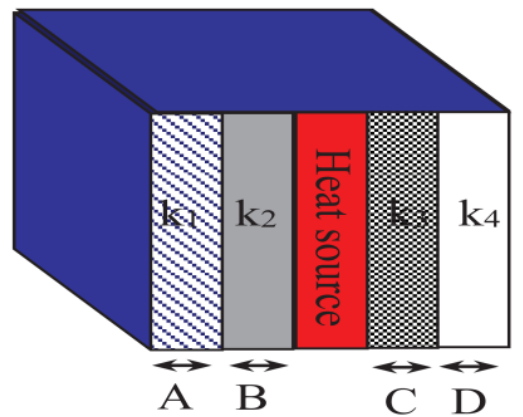

Fig.3. Heat source by insulating materials.

The material widths are not precise and they are in the form of Z-numbers.

\section{Conclusion}

engineers tend to deal with various types of measures as well as evaluations. However, all evaluation of information may not be presented as a clear number.

Z-number has considerable ability in explaining the vagueness of human knowledge. In this paper, the applications of Z-numbers in engineering are studied. The most current researches in the area of Z-numbers are covered in this work. Since some engineers have successfully applied Z-numbers, detailed discussions are supplied to stimulate future investigations.

\section{References}

1. Jafari, R., Razvarz, S., Gegov, A.: A New Computational Method for Solving Fully Fuzzy Nonlinear Systems, In: Computational Collective Intelligence. ICCCI 2018. Lecture Notes in Computer Science, Springer, Cham, 11055, 503-512 (2018).

2. Razvarz, S., Jafari, R., Gegov, A., Yu, W., Paul, S.: Neural network approach to solving fully fuzzy nonlinear systems, Fuzzy modeling and control Methods Application and Research, Nova science publisher, Inc, NewYork. ISBN: 978-1-53613415-5, 45-68 (2018).

3. Jafari, R., Razvarz, S.: Solution of Fuzzy Differential Equations using Fuzzy Sumudu Transforms, IEEE International Conference on Innovations in Intelligent Systems and Applications, 84-89, (2017).

4. Jafari, R., Razvarz, S.: Solution of fuzzy differential equations using fuzzy sumudu transforms, Mathematical and Computational Applications, 1-15, (2018).

5. Negoita, C.V., Ralescu, D.A.: Applications of Fuzzy Sets to Systems Analysis, Wiley, New York, (1975).

6. Zadeh, L.A.: Probability measures of fuzzy events, Journal of Mathematical Analysis and Applications, 23, 421-427, (1968). 
7. Zadeh, L.A.: Calculus of fuzzy restrictions, Fuzzy sets and Their Applications to CognitiveandDecision Processes, Academic Press, New York, 1975, 1-39.

8. Zadeh, L.A.: Fuzzy logic and the calculi of fuzzy rules and fuzzy graphs, MultipleValued Logic, 1, 1-38, (1996).

9. Jafari, R., Yu, W., Li, X.: Solving Fuzzy Differential Equation with Bernstein Neural Networks, IEEE International Conference on Systems, Man, and Cybernetics, Budapest, Hungary, 1245-1250 (2016).

10. Jafari, R. Yu, W.: Uncertain nonlinear system control with fuzzy differential equations and Z-numbers, 18th IEEE International Conference on Industrial Technology, Canada, 890-895, doi:10.1109/ICIT.2017.7915477, (2017).

11. Jafarian, A., Measoomy nia, S., Jafari, R.: Solving Fuzzy Equations Using Neural Nets with a New Learning Algorithm, Journal of Advances in Computer Research, 3, 33-45, (2012)

12. Jafarian, A., Jafari, R.: A new computational method for solving fully fuzzy nonlinear matrix equations, Int. J. Fuzzy Computation and Modelling, 2, 275-285, (2019).

13. Jafari, R., Razvarz, S., Gegov, A.: A novel technique to solve fully fuzzy nonlinear matrix equations, Advances in Intelligent Systems and Computing, Springer, $13^{\text {th }}$ International Conference on Applications of Fuzzy Systems and Soft Computing, Warsaw, Poland, (2018).

14. Zadeh, L.A.: A note on Z-numbers, Inform. Sciences. 181, 29232932, (2011).

15. Jafari, R., Razvarz, S., Gegov, A., Paul, S.: Fuzzy modeling for uncertain nonlinear systems using fuzzy equations and Z-numbers, Advances in Computational Intelligence Systems: Contributions Presented at the 18th UK Workshop on Computational Intelligence, September 5-7, 2018, Nottingham, UK . Advances in Intelligent Systems and Computing, Springer, 840, 66-107 (2018).

16. Jafari R., Razvarz S., Gegov A.: Solving Differential Equations with Z-Numbers by Utilizing Fuzzy Sumudu Transform, Intelligent Systems and Applications. IntelliSys 2018. Advances in Intelligent Systems and Computing, Springer, Cham. 869, 1125-1138, (2019).

17. Yu, W., Jafari, R.: Fuzzy Modeling and Control with Fuzzy Equations and Z- Number, IEEE Press Series on Systems Science and Engineering, Wiley-IEEE Press, ISBN-13: 978-1119491552, (2019).

18. Jiang, W., Xie, C., Luo, Y., Tang, Y.: Ranking Z-numbers with an improved ranking method for generalized fuzzy numbers, Journal of Intelligent and Fuzzy Systems, 32, 1931-1943, (2017).

19. Yaakob, A.M., Gegov, A.: Fuzzy rule based approach with Z-numbers for selection of alternatives using TOPSIS, Proceedings of the IEEE International Conference on Fuzzy Systems, 1-8, (2015).

20. Zamri, N., Ahmad, F., Rose, A.N.M., Makhtar, M.: A Fuzzy TOPSIS with Z- Numbers Approach for Evaluation on Accident at the Construction Site, Proceed- ings of the International Conference on Soft Computing and Data Mining, 41-50, (2016).

21. Razvarz, S., Jafari, R., Yu, W.: Numerical Solution of Fuzzy Differential Equations with Z-numbers using Fuzzy Sumudu Transforms, Advances in Science, Technology and Engineering Systems Journal (ASTESJ), 3, 66-75, doi: 10.25046/aj030108, (2018). 
22. Jafari, R., Razvarz, S., Gegov, A., Paul, S., Keshtkar, S.: Fuzzy Sumudu Transform Approach to Solving Fuzzy Differential Equations With Z-Numbers, Advanced Fuzzy Logic Approaches in Engineering Science, IGI Global, doi: 10.4018/978-15225-5709-8.ch002, (2018).

23. Azadeh, A., Saberi, M., Atashbar, N.Z., Chang, E., Pazhoheshfar, P.: Z- AHP: a Znumber extension of fuzzy analytical hierarchy process, Proc 7th IEEE Int Conf on Digital Ecosystems and Technologies, 141-147, https://doi.org/10.1109/DEST.2013.6611344, (2013).

24. Aliev, R.A., Alizadeh, A.V., Huseynov, O.H.: The arithmetic of discrete Z-numbers, Inform Sci, 290, 134-155, https://doi.org/10.1016/j.ins.2014.08.024, (2015).

25. Aliev, R.A., Huseynov, O.H., Zeinalova, L.M.: The arithmetic of continuous Znumbers, Inform Sci, 373, 441-460, https://doi.org/10.1016/j.ins.2016.08.078, (2016).

26. De Barros, L.C., Bassanezi, R.C., Lodwick, W.A.: The Extension Principle of Zadeh and Fuzzy Numbers, A First Course in Fuzzy Logic, Fuzzy Dynamical Systems, and Biomathematics. Studies in Fuzziness and Soft Computing, Springer, Berlin, Heidelberg, 347, 23-41, (2017).

27. Jiang, W., Xie, Ch., Zhuang, M., Shou, Y., Tang, Y.: Sensor Data Fusion with ZNumbers and Its Application in Fault Diagnosis, Sensors, 16, 1509; doi:10.3390/s16091509, (2016).

28. Jafari, R., Yu, W., Li, X., Razvarz, S.: Numerical solution of fuzzy differential equations with Z-numbers using bernstein neural networks, International Journal of Computational Intelligence Systems, 10, 1226-1237, (2017).

29. Nuriyev, A.M.: Application of Z-Numbers Based Approach to Project Risks Assessment, European Journal of Interdisciplinary Studies, 5, 67-73, (2019).

30. Jafari, R., Yu, W., Li, X.: Numerical solution of fuzzy equations with Z-numbers using neural networks, Intelligent automation and Soft Computing, 1-7, (2017). 